\title{
Composición y estructura de la macrofauna asociada con agregaciones de dos especies de bivalvos en Isla de Cubagua, Venezuela
}

\author{
Iván Hernández-Ávila ${ }^{1}$, Alejandro Tagliafico ${ }^{2} \&$ Nestor Rago $^{3}$ \\ 1. Departamento de Ciencias, Unidad de Cursos Básicos, Universidad de Oriente, Isla de Margarita, Venezuela; \\ ivanhernavila@yahoo.com \\ 2. Escuela de Ciencias Aplicadas del Mar, Universidad de Oriente, Isla de Margarita, Venezuela; tagliaficoa@gmail.com \\ 3. Oceanografía y pesca, Fundación de Ciencias Naturales La Salle, Isla de Margarita Venezuela; nstorago@gmail.com
}

Recibido 10-I-2012. Corregido 03-IX-2012. Aceptado 01-X-2012.

\begin{abstract}
Composition and structure of the macrofauna associated with beds of two bivalve species in Cubagua Island, Venezuela. Bivalve aggregations constitute a microhabitat for a wide variety of organisms in intertidal, subtidal and deep-water marine benthic habitats. Increase in density of bivalve beds could offer more crevices and substratum for the associated fauna, affecting community composition. Beds of the Atlantic Pearl Oyster (Pinctada imbricata) and the Turkey Wing (Arca zebra) of contrasting population densities were evaluated to determine the composition and structure of the associated macrofauna of three taxa (Crustacea Decapoda, Mollusca and Echinodermata). We evaluated plots of three levels of bed density in both species, the associated fauna were identified and counted. Other species were collected by qualitative samples. Univariate and multivariate descriptors were tested comparing the associated fauna between the beds of two species at three levels of density. In these beds a total of 104 species belonging to 58 families were recorded. Mithraculus forceps (Majidae), Crucibulum auricula (Calyptraeidae) and Ophiotrix angulata (Ophiothrichidae) were the most common species found in these assemblages. The medium and high-density bivalve beds exhibited greater species number, abundance, Shannon diversity, evenness, taxonomic diversity, and taxonomic distinctness of associated fauna, than low-density bivalve beds. Moreover, multivariate analysis detected different assemblages of associated fauna between beds with different densities. Additionally, similarities were found in the communities of macrofauna in both beds of $P$. imbricata and Arca zebra. Our results suggest that bivalve aggregations at Cubagua Island provide additional habitat for macrofauna living in other shallow habitats such as Thalassia beds, corals and rocky environments. Bed density, associated with topographic complexity, represents an important factor for the composition and complexity of the associated fauna. Rev. Biol. Trop. 61 (2): 669-682. Epub 2013 June 01.
\end{abstract}

Key words: Crustacea, Mollusca, Echinodermata, Arca zebra, Pinctada imbricata, associated fauna.

Las agregaciones de bivalvos constituyen un microhábitat para una gran variedad de organismos en el ambiente intermareal, submareal y en aguas profundas (Tsuchiya $\&$ Nishihira 1985, 1986, Thiel \& Ulrich 2002, Turnipseed et al. 2004, Galkin \& Goroslavskaya 2008). Estas agregaciones incrementan la heterogeneidad espacial del ambiente bentónico y proveen refugio y alimento para una gran diversidad de organismos (Jacobi 1987, Seed 1996, Jones et al. 1997). La diversidad local, las tramas alimenticias y los ciclos de nutrientes pueden verse afectados por la presencia de agregaciones de bivalvos, que usualmente incrementan la abundancia y diversidad de la fauna asociada (Tsuchiya \& Nishihira 1985, Thiel \& Ulrich 2002, Gutierrez et al. 2003, Carranza et al. 2009a, 2009b).

En el sureste del Caribe, agregaciones conspicuas de Arca zebra y de la ostra perla (Pinctada imbricata) están presentes en ambientes marinos de aguas someras. La ostra perla ha sido explotada para la producción de perlas desde el siglo XV (Otte 1977, Cervigón 
1997, 1998, Mackenzie et al. 2003). Actualmente, soporta una pequeña pesquería artesanal, principalmente para consumo local como alimento, mientras que $A$. zebra soporta una pesquería artesanal mayor, localmente importante, con un volumen entre $35000-40000 \mathrm{tm} /$ año, el cual varía dependiendo de la demanda de la industria enlatadora (Arias et al. 2002). Ambas especies tienen una amplia distribución y forman agregaciones con fauna bentónica asociada (Prieto et al. 2001a, Díaz \& Liñero 2003, Licet et al. 2009).

La fauna asociada con agregaciones de bivalvos de diferentes especies ha sido estudiada al comparar gradientes espaciales grandes (Thiel \& Ulrich 2002), y diferentes tamaños de agregaciones de bivalvos (Tsuchiya \& Nishihira 1985), y al incorporar otros factores como la edad, tamaño y densidad de los parches (Tsuchiya \& Nishihira 1986, Borthagaray \& Carranza 2007). También se han comparado agregaciones de diferentes especies en áreas separadas (Turnipseed et al. 2004), o a diferentes profundidades (Van Dover \& Trask 2000). Sin embargo, estudios sobre las diferencias en macrofauna asociada con agregaciones de diferentes especies en la misma área son escasos y la mayoría de los estudios en fauna asociada a bivalvos han sido realizados en aguas templadas. El papel de las agregaciones de bivalvos en ambientes bentónicos tropicales ha sido poco estudiado (Prieto et al. 2001b, Villafranca \& Jiménez 2004, 2006, Licet et al. 2009).

Múltiples trabajos han mostrado la importancia de la complejidad topográfica en la estructura de la fauna bentónica (Bourget et al. 1994, Chapman \& Underwood 1994, Pech et al. 2001). El número de especies, la abundancia y la diversidad de las comunidades bentónicas están positivamente correlacionadas con la complejidad topográfica (Gutiérrez et al. 2003, Borthagary \& Carranza 2007). El incremento en la densidad poblacional de las agregaciones de bivalvos ha sido asociado con una mayor complejidad del sustrato, pudiendo ofrecer mayor cantidad de grietas, elevaciones y espacio disponible para la fauna asociada, disminuyendo la competencia inter e intraespecífica por espacio, se presenta así una relación positiva con la abundancia, número de especies y diversidad. En el presente estudio, ponemos a prueba la hipótesis de que la abundancia, diversidad y estructura de la macrofauna asociada con agregaciones de bivalvos, cambia con relación a la densidad poblacional de los bivalvos. Esta hipótesis se pone a prueba en agregaciones de dos especies simpátricas de bivalvos en un ambiente tropical (Isla de Cubagua, Venezuela), se evalúa la respuesta de los táxones Crustacea Decapoda, Mollusca y Echinodermata.

\section{MATERIALES Y MÉTODOS}

Área de estudio: La isla de Cubagua es de naturaleza semidesértica, posee alrededor de $22 \mathrm{~km}^{2}$ y está situada al noreste de Venezuela ( $10^{\circ} 47^{\prime}$ y $10^{\circ} 51^{\prime} \mathrm{N}-64^{\circ} 8^{\prime}$ y $\left.64^{\circ} 14^{\prime} \mathrm{W}\right)$. La zona está fuertemente influenciada por el sistema de surgencia costera estacional y caracterizada por una plataforma continental somera con fondos arenosos compuestos principalmente por sedimento grueso (Cervigón 2005). El proceso de surgencia ocurre principalmente durante los primeros meses del año (Enero-Mayo), y consiste principalmente en la intrusión estacional de aguas con bajas temperaturas, oxígeno disuelto y elevados nutrientes (Gómez 1996). La temperatura superficial del agua varía entre $22-26^{\circ} \mathrm{C}$ y exhibe una elevada productividad (Gómez 1996). El litoral de la isla está compuesto principalmente de aguas someras (0-10m de profundidad) con fondos compuestos por arrecifes marginales, costa rocosa, praderas de Thalassia testudinum, agregaciones de A. zebra y $P$. imbricata y ambientes arenosos (Cervigón 2005). En la región sureste de la isla, se encuentran agregaciones conspicuas de $A$. zebra y P. imbricata entre los 200 a $500 \mathrm{~m}$ de la línea de costa. Estudios previos de la fauna bentónica de la isla han estado enfocados a inventarios y distribución de especies (Capelo \& Buitrago 1998, Hernández-Ávila et al. 2007, Hernández-Ávila 2010, Tagliafico et al. 2011). 
Toma de muestras: La estructura de la macrofauna asociada de crustáceos decápodos, moluscos y equinodermos fue comparada entre agregaciones de bivalvos de diferentes especies así como entre diferentes densidades poblacionales de estos. La comparación entre especies que forman las agregaciones se realizó a dos niveles ( $P$. imbricata y A. zebra), y el efecto de la densidad poblacional de las agregaciones fue estudiado considerando tres niveles discretos de abundancia de bivalvos que componen la agregación (bajo: 60-100; medio: 100-200; alto: mayor a 200 ind. $/ \mathrm{m}^{2}$ ), conformando un diseño factorial de dos vías con siete réplicas aleatorias por combinación. Las muestras fueron recolectadas entre Septiembre 2005-Mayo 2006 durante periodos sin surgencia (Septiembre-Noviembre) y con surgencia (diciembremayo). Para excluir el efecto potencial de los periodos de muestreo en el diseño, las réplicas se distribuyeron aleatoriamente entre los periodos. Aunque la aleatorización puede generar patrones adicionales en diseño experimental (Hurlbert 1984), no fueron detectados patrones de muestreo entre los periodos $\left(\chi^{2} \mathrm{r} x \mathrm{c}\right.$ test $=9.17, \mathrm{df}=5, \mathrm{p}=0.12$ ).

Las muestras fueron recolectadas en parcelas de $0.5 \times 0.5 \mathrm{~m}$ distribuidas al azar, utilizando equipo de buceo autónomo a profundidades entre $5-12 \mathrm{~m}$. Las parcelas fueron cubiertas con bolsas plásticas y todos los bivalvos y sus respectivos componentes bentónicos asociados fueron removidos cuidadosamente de forma que fuesen contenidos en las bolsas plásticas y evitar el escape de organismos móviles. Posteriormente, las muestras fueron fijadas para su evaluación en el laboratorio. Los bivalvos que forman las agregaciones fueron contados para verificar el nivel de densidad y la prevalencia de la especie que forma la agregación. Se omitieron parcelas caracterizadas por bancos mixtos (con proporción similar de $P$. imbricata y $A$. zebra) debido a que representaron pocos casos y no estaban contemplados en el diseño. Los componentes de la macrofauna asociada fueron separados y fijados con etanol al 95\%, para su identificación y cuantificación. Se realizaron muestreos cualitativos adicionales en agregaciones de ambas especies con equipo autónomo para identificar especies que no fueron detectadas en los muestreos cuantitativos.

Todos los ejemplares de moluscos, crustáceos decápodos y equinodermos fueron contados, se les determinó el número de especies (S), la abundancia total $(\mathrm{N})$, la equidad (J') (Pielou 1975), la diversidad de Shannon (H') (Shannon 1948), la diversidad taxonómica $(\Delta)$ y la distinción taxonómica $\left(\Delta^{*}\right)$ (Warwick \& Clark 1995), como descriptores univariados de cada muestra. En relación a la distinción y diversidad taxonómica, se consideraron seis niveles de jerarquía: Phylum, Clase, Orden, Familia, Género y Especie. La clasificación de los grupos sigue el criterio de Díaz \& Puyana (1994) y Rosenberg (2009) para moluscos, De Grave et al. (2009) para crustáceos decápodos y Hendler et al. (1995) para equinodermos. Especímenes de referencia fueron catalogados y conservados en la colección del Laboratorio del Departamento de Ciencias de la Universidad de Oriente, Núcleo de Nueva Esparta.

El número de especies, la abundancia total, la equidad, la diversidad de Shannon, la diversidad taxonómica y la distinción taxonómica fueron contrastados entre diferentes densidades y especies de bivalvos utilizando un análisis de varianza permutado (PERMANOVA) (Anderson 2001, McArdle \& Anderson 2001), con base en el índice de similitud de las Distancias Euclidianas. Se probó la hipótesis nula de no diferencias en los descriptores univariados (S, $\mathrm{N}, \mathrm{J}$ ', H', $\Delta, \Delta^{*}$ en cada caso) asociado con los factores densidad y especie de bivalvo, además de su interacción. Pruebas $t$ permutada fueron utilizadas como análisis a posteriori para detectar grupos entre los niveles de densidad.

Para probar la hipótesis nula de no diferencias en la estructura del ensamble de la macrofauna asociado a los factores anteriormente descritos, se construyó una matriz de similitud utilizando el índice de Bray-Curtis y fue probada la hipótesis con un análisis PERMANOVA. Siguiendo lo recomendado por Clarke et al. (2006) se adicionó una variable adicional con valor igual a uno para todas las muestras. El número de permutaciones de los residuales 
utilizado para determinar la significancia estadística de cada término fue 999 bajo el modelo reducido (Anderson 2001). Una prueba de dispersión multivariada (PERMDISP) fue utilizada para identificar si los componentes de variación significativa estuvieron relacionados con diferencias en la dispersión multivariada o con diferencias en la posición de los centroides. Un análisis SIMPER (Clarke 1993) fue realizado para identificar a las especies asociadas con diferencias entre los grupos, cuando fueron detectadas.

\section{RESULTADOS}

Se identificaron 104 especies distribuidas en 58 familias, 39 especies de crustáceos decápodos, 46 moluscos y 19 equinodermos (Apéndice 1), 92 especies fueron recolectadas durante los muestreos cuantitativos, mientras las especies adicionales fueron identificadas a partir de muestras cualitativas adicionales. Acorde con su presencia y abundancia, las especies más frecuentes fueron: Mithraculus forceps (Majidae), Crucibulum auricula (Calyptraeidae) y Ophiotrix angulata (Ophiothrichidae).

No fueron detectadas diferencias en los descriptores univariados entre la fauna asociada con agregaciones $P$. imbricata y A. zebra. Sin embargo, el número de especies, la abundancia total, la diversidad, la equidad, la diversidad taxonómica y la distinción taxonómica mostraron diferencias significativas asociadas a la densidad de las agregaciones (Cuadro 1). El componente de variación asociado a la densidad del banco varió entre 27.6 y $65.3 \%$ de la variación total para los índices univariados. Mientras que la variación asociada a la especie que forma el bando varió entre 0 y $2.2 \%$ y no fue significativa en todos los casos.

Además del efecto asociado a la densidad de los bivalvos se detectó un componente de variación asociado a los residuales que varió entre 33.3 y $65.8 \%$. Esta variación corresponde a las diferencias existentes entre las réplicas de cada combinación, las cuales estaban separadas en escalas menores a $100 \mathrm{~m}$. Lo cual sugiere una alta variación a escala espacial pequeña dentro de cada nivel de densidad.

La prueba $t$ aplicada a los niveles del factor densidad, mostró en todos los casos que las agregaciones con densidad baja fueron diferentes a las agregaciones de densidad media y alta, los dos últimos niveles no mostraron diferencias significativas excepto para abundancia total que presentó diferencias significativas entre los tres niveles de densidad. Los niveles de densidad media y alta de las agregaciones mostraron una fauna asociada con número mayor de especies $(S=10.18 \pm 0.72$, media \pm error estándar), abundancia $\left(\mathrm{N}=28.86 \pm 3.01 \mathrm{ind} / 0.25 \mathrm{~m}^{2}\right.$ D. Media, $39.43 \pm 3.54 \mathrm{ind} / 0.25 \mathrm{~m}^{2}$ D. Alta), diversidad de Shannon $\left(H^{\prime}=1.82 \pm 0.10 \mathrm{bits} /\right.$ ind), equidad $\left(\mathrm{J}^{\prime}=0.81 \pm 0.10\right)$, diversidad taxonómica $(\Delta=65.49 \pm 3.97)$ y distinción taxonómica $\left(\Delta^{*}=83.04 \pm 3.64\right)$; en comparación con la fauna asociada a agregaciones de bivalvos con densidad baja $(\mathrm{S}=3.36 \pm 0.94$, $\mathrm{N}=8.43 \pm 2.07 \mathrm{ind} / 0.25 \mathrm{~m}^{2}, \quad \mathrm{H}^{\prime}=0.67 \pm 0.23$ bits $/$ ind, $\quad \mathrm{J}^{\prime}=0.39 \pm 0.13, \quad \Delta=31.83 \pm 10.31$, $\Delta^{*}=36.68 \pm 11.75$ ) (Fig. 1).

El análisis de varianza multivariado permutado (PERMANOVA) mostró diferencias entre la composición de la fauna asociada a diferentes densidades de las agregaciones, con un componente de variación asociado a este factor de $15 \%$. Sin embargo, no se detectaron diferencias entre la fauna asociada de $P$. imbricata y de $A$. zebra, ni en su interacción con el factor densidad (Cuadro 2). En el factor densidad no fueron detectadas diferencias en la dispersión multivariada (PERMDISP, $\mathrm{F}=3.185$, Pseudo- $\mathrm{p}=0.076$ ).

Los ensambles de la macrofauna presente en agregaciones de baja densidad mostraron ser diferentes a aquellos ubicados en agregaciones con densidades media y alta $(\mathrm{t}=1.782, \mathrm{p}=0.003$; y $\mathrm{t}=2.450, \mathrm{p}=0.001$ respectivamente); además, los niveles de densidad media y alta también mostraron diferencias significativas $(\mathrm{t}=1.410$, $\mathrm{p}=0.026$ ), por lo que los tres niveles mostraron ser diferentes entre sí (Fig. 2). De acuerdo con el análisis SIMPER las especies que contribuyeron en mayor proporción a las diferencias en la composición de la fauna entre los niveles 


\section{CUADRO 1}

Análisis de la varianza permutado (PERMANOVA) de los indicadores univariados riqueza, abundancia, Shannon, equidad, diversidad taxonómica y distinción taxonómica. \%CV porcentaje de los componentes de variación asociada

TABLE 1

Permutated analysis of variance (PERMANOVA) of univariate descriptors: richness, abundance, Shannon, evenness, taxonomic diversity, taxonomic distinctness. $\% \mathrm{CV}$ percent estimates of components of variation

\begin{tabular}{|c|c|c|c|c|c|}
\hline Fuente de Variación & $\mathrm{gl}$ & Pseudo-F & $\mathrm{p}($ perm $)$ & perms & $\% \mathrm{CV}$ \\
\hline \multicolumn{6}{|l|}{ Riqueza } \\
\hline Banco & 1 & 0.355 & 0.559 & 997 & 0.0 \\
\hline Densidad & 2 & 16.828 & 0.001 & 999 & 47.40 \\
\hline Banco x Densidad & 2 & 2.567 & 0.076 & 999 & 9.39 \\
\hline Residual & 36 & & & & 41.93 \\
\hline \multicolumn{6}{|l|}{ Abundancia } \\
\hline Banco & 1 & 0.412 & 0.530 & 994 & 0.93 \\
\hline Densidad & 2 & 28.458 & 0.001 & 999 & 65.29 \\
\hline Banco x Densidad & 2 & 1.105 & 0.337 & 999 & 0.50 \\
\hline Residual & 36 & & & & 33.29 \\
\hline \multicolumn{6}{|l|}{ Shannon } \\
\hline Banco & 1 & 1.381 & 0.252 & 996 & 0.91 \\
\hline Densidad & 2 & 14.436 & 0.001 & 998 & 48.34 \\
\hline Banco x Densidad & 2 & 1.518 & 0.227 & 999 & 0.37 \\
\hline Residual & 36 & & & & 50.37 \\
\hline \multicolumn{6}{|l|}{ Equidad } \\
\hline Banco & 1 & 0.982 & 0.308 & 996 & 0.49 \\
\hline Densidad & 2 & 9.827 & 0.002 & 998 & 36.56 \\
\hline Banco x Densidad & 2 & 0.938 & 0.404 & 998 & 5.16 \\
\hline Residual & 36 & & & & 57.79 \\
\hline \multicolumn{6}{|l|}{ Diversidad Tax. } \\
\hline Banco & 1 & 1.699 & 0.201 & 997 & 2.19 \\
\hline Densidad & 2 & 6.877 & 0.005 & 997 & 27.63 \\
\hline Banco x Densidad & 2 & 1.463 & 0.236 & 999 & 4.36 \\
\hline Residual & 36 & & & & 65.82 \\
\hline \multicolumn{6}{|l|}{ Distinción Tax. } \\
\hline Banco & 1 & 1.358 & 0.237 & 995 & 0.96 \\
\hline Densidad & 2 & 11.477 & 0.001 & 998 & 42.18 \\
\hline Banco x Densidad & 2 & 1.062 & 0.359 & 999 & 0.50 \\
\hline Residual & 36 & & & & 56.36 \\
\hline
\end{tabular}

CUADRO 2

PERMANOVA de similitud de los ensambles de macrofauna, basado en el índice de Bray-Curtis. $\% \mathrm{CV}$ porcentaje de los componentes de variación asociada

TABLE 2

PERMANOVA of similarity of macrofauna assemblage, based on Bray-Curtis Index. $\% \mathrm{CV}$ percent estimates of components of variation

\begin{tabular}{lccccc}
\multicolumn{1}{c}{ Fuente de Variación } & gl & Pseudo-F & P(perm) & perms & $\% \mathrm{CV}$ \\
Banco & 1 & 1.390 & 0.166 & 999 & 1.54 \\
Densidad & 2 & 3.648 & 0.001 & 999 & 15.66 \\
Banco x Densidad & 2 & 1.0 & 0.491 & 998 & 0.00 \\
Residual & 36 & & & 82.80 \\
\hline
\end{tabular}



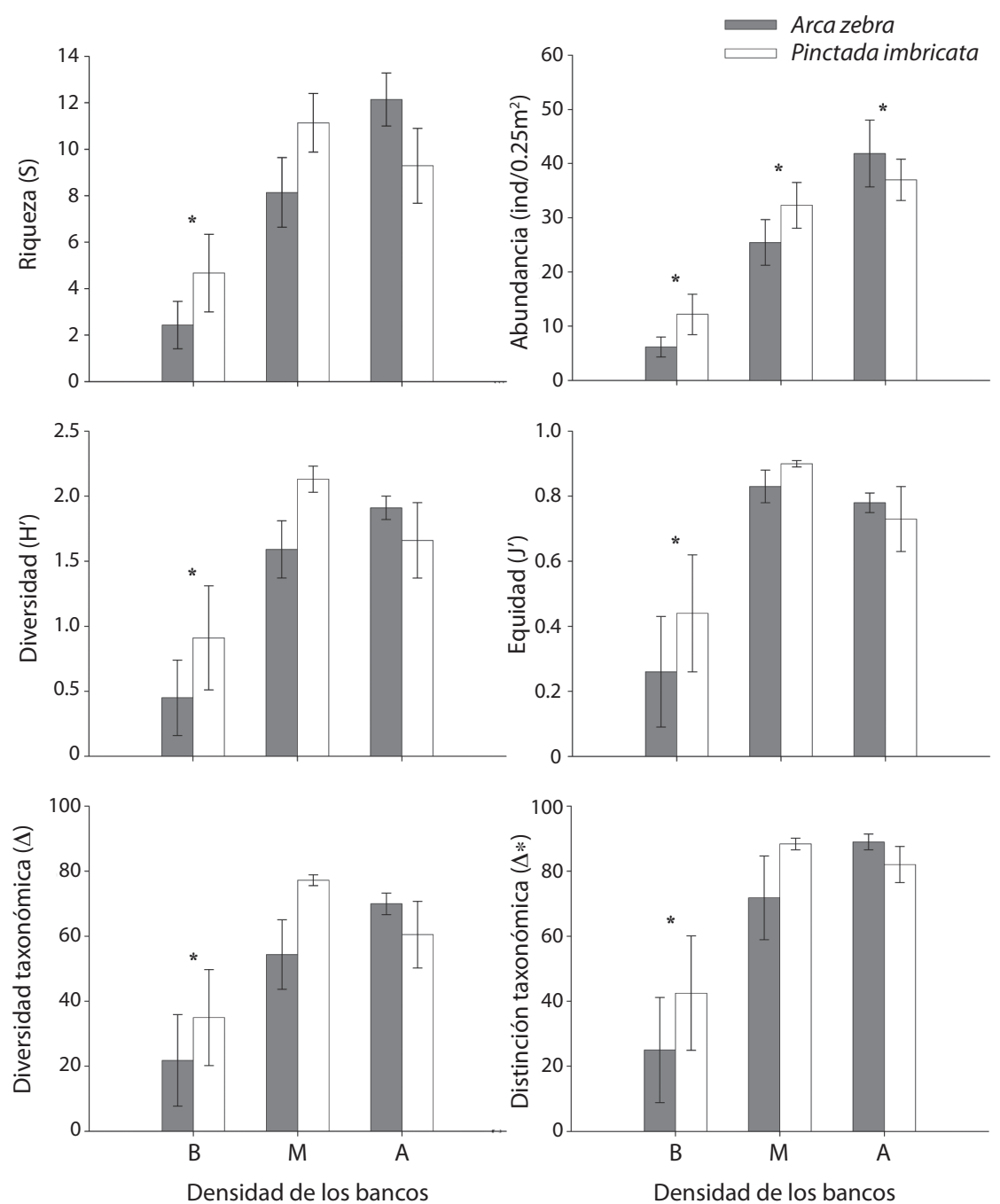

Fig. 1. Indicadores comunitarios de crustáceos decápodos, moluscos y equinodermos asociados a bancos de $P$. imbricata y A. zebra en función a los niveles de densidad de los bancos de bivalvos. $\mathrm{B}=$ densidad baja $\left(60-100\right.$ ind. $\left./ \mathrm{m}^{2}\right), \mathrm{M}=\mathrm{densidad}$ media $\left(100-200\right.$ ind. $\left./ \mathrm{m}^{2}\right), \mathrm{A}=$ densidad alta $\left(200 \mathrm{ind} . / \mathrm{m}^{2}\right) .{ }^{*}$ Diferencias significativas $(t, \mathrm{p}<0.05)$ en niveles de densidad.

Fig. 1. Community index of decapod crustaceans, mollusks and echinoderms associated to beds of P. imbricata and A. zebra in relation with levels of density of bivalve beds. $B=$ low density $\left(60-100 \mathrm{ind} . / \mathrm{m}^{2}\right), \mathrm{M}=$ medium density $\left(100-200 \mathrm{ind} . / \mathrm{m}^{2}\right)$, $\mathrm{A}=$ high density $\left(200 \mathrm{ind} . / \mathrm{m}^{2}\right) .{ }^{*}$ Significant differences $(t, \mathrm{p}<0.05)$ in density levels.

de densidad fueron: Mithraculus forceps (contribución a la disimilaridad 20.66\%), Ophiotrix angulata (12.59\%), Crucibulum auricula (11.49\%) y Chama floridana (5.82\%). La contribución del resto de las especies a la disimilitud fue menor al 5\%. En todos los casos, el patrón de abundancia de las especies que contribuyeron a la varianza entre grupos de densidad presentó un gradiente de incremento, desde las agregaciones con densidad bajas hacia las agregaciones con densidades altas.

\section{DISCUSIÓN}

Las diferencias detectadas en la fauna asociada en relación al factor densidad poblacional de los bivalvos demuestran que las 


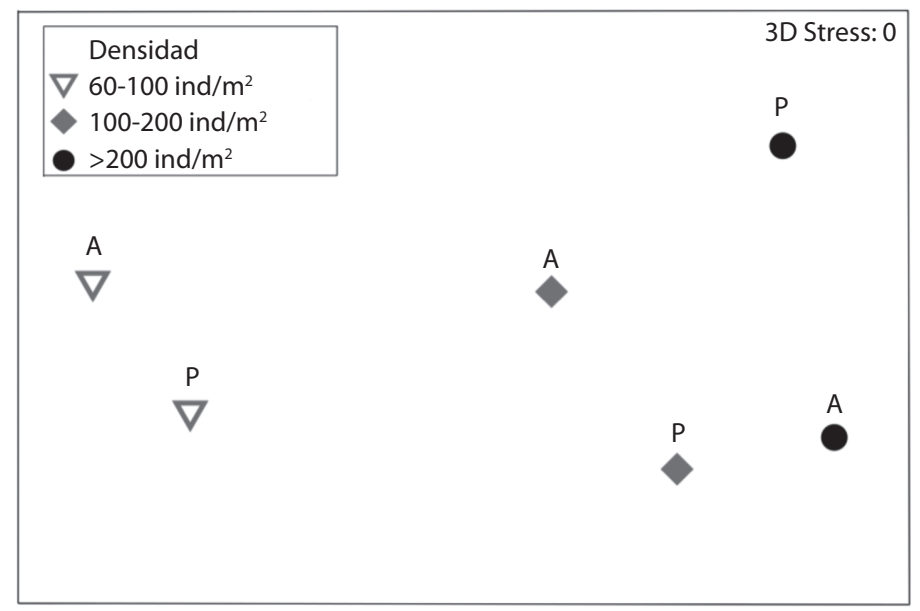

Fig. 2. Escalamiento multidimensional no métrico de los centroides para las combinaciones densidad y bivalvo que forma el banco, con base al índice de Bray-Curtis. $\mathrm{P}=$ bancos de P. immbricata, $\mathrm{A}=$ bancos de A. zebra.

Fig. 2. No-metric mutidimentional scaling centroids of combinations of density and bivalve beds, based in Bray-Curtis Index. . $\mathrm{P}=$ P. imbricata beds, $\mathrm{A}=A$. zebra beds.

comunidades asentadas en agregaciones densas de bivalvos son más complejas, al mostrar mayor riqueza, abundancia y equidad, además de mayor diversidad y distinción taxonómica. Estos dos últimos indicadores permiten deducir que las asociaciones de macrofauna en agregaciones densas de bivalvos están comprendidas por especies de diversos grupos taxonómicos, mientras que la macrofauna en agregaciones poco densas está comprendida por especies que pertenecen a pocos grupos taxonómicos. Las especies caracterizaron los bancos de baja densidad fueron: $M$. forceps, $O$. angulata, $C$. auricula y $C$. florida; a medida que se incrementa la densidad de los bivalvos se incorporan nuevas especies aumentando la riqueza. Adicionalmente la estructura taxonómica general de la fauna asociada presentó pocas especies por familia, por lo que el incremento de especies en bancos densos usualmente involucraba la adición de nuevas familias, incrementando la diversidad taxonómica del ensamble.

Se ha mencionado que los bivalvos que forman agregaciones pueden funcionar como bioingenieros al crear condiciones para el mantenimiento de algas y animales en ambientes costeros así como en aguas profundas (Turnipseed et al. 2004, Beck et al. 2009, 2011). La tendencia general sobre estas agregaciones es que la diversidad y abundancia de la macrofauna en ellas es mayor que en el ambiente circundante (Gutiérrez et al. 2003), aunque se han observado excepciones de esta tendencia (Buschbaum et al. 2009). Entre los roles identificados para agregaciones de bivalvos se mencionan la reducción de la materia suspendida y de nutrientes en sedimentos (Newell \& Koch 2004), así como la generación de un sustrato duro y heterogéneo que sirve de hábitat para múltiples especies, algunas de las cuales depredan los bivalvos (Gutiérrez et al. 2003). El número y composición de especies asociadas a los bivalvos en el presente estudio, demuestran que estos generan condiciones para el establecimiento de comunidades diferentes al entorno arenoso circundante, ajustándose al concepto de bioingenieros. Aunque queda por determinarse cuáles son los roles que controlan el desarrollo de las comunidades asociadas.

El incremento en la densidad de las agregaciones de bivalvos puede asociarse a un aumento en la complejidad topográfica del sustrato. Ambientes espacialmente complejos tienden a soportar comunidades faunísticas más ricas y complejas, posiblemente debido a que proveen mayor número de cavidades 
(Abele 1974, Ricciardi et al. 1997). Entre las propiedades asociadas al incremento en la complejidad topográfica se mencionan el aumento del sustrato disponible, el número de nichos y modos de explotar recursos y la disminución en la efectividad de los depredadores (MacArthur \& MacArthur 1961, Russo, 1987, Matias et al. 2010). Estas propiedades están asociadas al incremento en la riqueza y complejidad de las comunidades, y han sido señaladas en agregaciones densas de bivalvos.

Se ha indicado que bancos de bivalvos poseen gran cantidad de espacios intersticiales que sirven como refugio contra la predación para macrofauna móvil (Gosselin \& Chia 1995, Borthagaray \& Carranza 2007). Además el incremento en la complejidad estructural en agregaciones densas puede disminuir la competencia por el espacio entre miembros de la comunidad, ya que supone el incremento en el área total disponible y en la formación de mayor número de microhábitats (Crooks \& Khim 1999, Guiterrez et al. 2003). Otros efectos asociados al incremento en las densidades de las agregaciones es el control de transporte de partículas (Gutiérrez et al. 2003), lo cual pudiera brindar un soporte trófico a las comunidades asociadas.

Además de la variación asociada a la densidad se encontró un porcentaje alto de la variación asociada a las réplicas existentes entre cada combinación, las cuales estaban separadas a una escala menor a $100 \mathrm{~m}$. Existen varios factores que pudieran generar esta variación, por ejemplo el tamaño de las parcelas o la variación en densidad de bivalvos dentro de cada categoría. Sin embargo no se descarta que estos sistemas presenten altas variaciones a pequeña escala, como ha sido señalado para bancos de bivalvos (Lintas \& Seed 1994, Seed 1996, Erlandsson et al. 2005), así como para otros sistemas costeros en la región (Cruz-Motta 2007, Guerra-Castro et al. 2011, Fuentes 2012).

No fueron detectadas diferencias en la macrofauna asociada con agregaciones de $A$. zebra y $P$. imbricata, lo cual sugiere que funcionan entre sí como hábitats alternativos. Los miembros de la macrofauna identificados en el presente estudio han sido señalados previamente en ambientes rocosos, praderas de Thalasia testudinum, y en ambientes arenosos, tanto en la isla de Cubagua como en otras localidades de Venezuela (Zoppi 1967, Rodríguez 1980, Capelo \& Buitrago 1998, Hernández-Ávila et al. 2007, Hernández-Ávila 2010, Tagliafico et al. 2011). Aunque algunas de las especies señaladas son poco frecuentes o han sido reportadas para la región recientemente (Hernández-Ávila et al. 2007), no se detectaron especies que presentaran asociación exclusiva con las agregaciones de bivalvos estudiadas.

Trabajos previos han identificado el papel de agregaciones de bivalvos como hábitat potencial o como refugio para fauna bentónica (Ruiz et al. 1993, Dittel et al. 1995, Eggleston 1998, Posey et al. 1999). La estructura física de las agregaciones de bivalvos ha mostrado servir como sustrato para el reclutamiento de invertebrados marinos tanto en experimentos de laboratorio como en el campo (Welch et al. 1997, Eggleston et al. 1998). Además se ha demostrado la selección activa de agregaciones de bivalvos por parte de camarones en respuesta a la presencia de depredadores (Posey et al. 1999). La presencia de múltiples hábitats alternativos para el reclutamiento y refugio pudiera favorecer el mantenimiento de las poblaciones en el bentos (Eggleston et al. 1998, 1999). Las similitudes de las comunidades entre los bancos de diferentes especies, así como la distribución de las especies asociadas en otros hábitats aledaños sugieren que la persistencia local de la fauna es favorecida por la existencia de múltiples hábitats para el refugio y el reclutamiento de organismos.

Actualmente, las agregaciones de ambas especies (A. zebra y P. imbricata) son explotadas por la pesquería artesanal, A. zebra está sometida a mayor explotación para el procesamiento industrial, además de poseer mayor comercialización que $P$. imbricata. Numerosos esfuerzos han sido dirigidos al estudio de la biología de ambas especies, con el fin de desarrollar condiciones de cultivo y para determinar algunos lineamientos relacionados 
a su extracción (Jiménez et al. 2000, Prieto et al. 2001b, Lodeiros et al. 2002, Marcano et al. 2005). De hecho, existen tanto vedas temporales como tallas mínimas de capturas para regular la pesca de ambos recursos (Novoa et al. 1998), sin embargo se observa que existen fallas en el cumplimiento de estas regulaciones y no se aplican mecanismos de vigilancia y control. La gran cantidad de fauna asociada reportada para ambas especies en estudios previos (Prieto et al. 2001a, Díaz \& Liñero 2003, Licet et al. 2009) y en el presente, así como el efecto de la densidad de las agregaciones de moluscos en la estructura de la macrofauna, sugiere la importancia del control adecuado de estas pesquerías, para garantizar el mantenimiento de sus densidades y con ellas las comunidades bentónicas asociadas.

\section{AGRADECIMIENTOS}

Los pescadores de Punta de Piedras colaboraron en el traslado a las zonas de extracción de moluscos. Jesús Marcano y Hargadis Zanabria ayudaron en la logística de los muestreos. Este trabajo fue parcialmente financiado por INIA y CIUDONE. Los comentarios editoriales y de los árbitros ayudaron a incrementar la calidad del trabajo.

\section{RESUMEN}

Las agregaciones de bivalvos constituyen un microhábitat para una gran variedad de organismos en el ambiente intermareal, submareal y en aguas profundas. Agregaciones de la ostra perla (Pinctada imbricata) y pepitona (Arca zebra) a diferentes densidades poblacionales se evaluaron para determinar la composición y estructura comunitaria de la macrofauna asociada en tres taxa (Crustacea Decapoda, Mollusca y Echinodermata). La hipótesis nula de no diferencias en descriptores univariados y multivariados fue probada comparando la fauna asociada entre las agregaciones de las dos especies a tres niveles de densidad. En estas agregaciones se identificaron 102 especies de 55 familias. Mithraculus forceps (Majidae), Crucibulum auricula (Calyptraeidae) y Ophiotrix angulata (Ophiothrichidae) fueron las especies más comunes encontradas en estas asociaciones. Las densidades medias y altas de las agregaciones de bivalvos presentaron mayor número de especies, abundancia, diversidad de Shannon, equidad, diversidad taxonómica y distinción taxonómica de la fauna asociada que las agregaciones de baja densidad poblacional. Análisis multivariados detectaron diferentes estructuras de los ensambles de la fauna asociada en agregaciones de bivalvos con densidad baja en comparación con los de densidad media y alta. Adicionalmente no se detectaron diferencias en la fauna asociada entre las especies. La densidad de las agregaciones de bivalvos, asociada a la complejidad topográfica, es un factor importante para la composición de la fauna asociada.

Palabras clave: crustáceos, moluscos, equinodermos, Pinctada imbricata, Arca zebra.

\section{REFERENCIAS}

Abele, L. 1974. Species diversity of Decapoda crustaceans in marine habitats. Ecology 55: 156-161.

Anderson, M.J. 2001. A new method for non-parametric multivariate analysis of variance. Austral Ecol. 26: 32-46.

Arias, A., R. Guzmán, R. Jiménez \& R. Molinet. 2002. La pesquería de la pepitona, Arca zebra, en Chacopata, estado Sucre, Venezuela: Un análisis bioeconómico. Zoot. Trop. 20: 49-67.

Beck, M.B., R.D. Brumbaugh, L. Airoldi, A. Carranza, L.D. Coen, C. Crawford, O. Defeo, G.J. Edgar, B. Hancock, M. Kay, H. Lenihan, M.W. Luckenbach, C.L. Toropova \& G. Zhang. 2009. Shellfish Reefs at Risk: A Global Analysis of Problems and Solutions. TNC, Arlington, EE.UU.

Beck, M.B., R.D. Brumbaugh, L. Airoldi, A. Carranza, L.D. Coen, C. Crawford, O. Defeo, G.J. Edgar, B. Hancock, M. Kay, H. Lenihan, M.W. Luckenbach, C.L. Toropova, G. Zhang \& X. Guo. 2011. Oyster reefs at risk and recommendation for conservation, restoration, and management. Bioscience 61: 107-116.

Borthagary, A.I. \& A. Carranza 2007. Mussels as ecosystem engineers: Their contribution to species richness in a rocky littoral community. Acta Oecol. 31: 243-250.

Bourget, E., J. De Guise \& G. Daigle. 1994. Scales of substratum heterogeneity, structural complexity, and early establishment of a marine epibenthic community. J. Exp. Mar. Biol. Ecol. 181: 31-51.

Buschbaum, C., S. Dittmann, J. Hong, I. Hwang, M. Strasser, M. Thiel, N. Valdivia, S. Yoon \& K. Reise. 2009. Mytilid mussels: global habitat engineering in coastal sediments. Helgoland Mar. Res. 63: 47-58.

Capelo, J.C. \& J. Buitrago. 1998. Distribución geográfica de los moluscos marinos en el oriente de Venezuela. Mem. Soc. Cs. Nat. Salle 58: 109-159.

Carranza, A, O. Deffeo \& M. Beck. 2009a. Diversity, conservation status and threats to native oysters 
(Ostreidae) around the Atlantic and Caribbean coasts of South America. Aquat. Conserv. 19: 344-353.

Carranza, A., O. Deffeo, M. Beck \& J.C. Castilla. 2009b. Linking fisheries management and conservation in bioengineering species: the case of South American mussels (Mytilidae). Rev. Fish Biol. Fisheries 19: 349-366.

Cervigón, F. 1997. Cubagua 500 años. Museo del Mar, Caracas, Venezuela.

Cervigón, F. 1998. Las perlas en la historia de Venezuela. Museo del Mar, Caracas, Venezuela.

Cervigón, F. 2005. Estudio bionómico de la isla de Cubagua. Informe FONACIT, Caracas, Venezuela.

Chapman, M.G. \& A.J. Underwood 1994. Dispersal of the intertidal snail, Nodolittorina pyramidalis, in response to the topographic complexity of the substratum. J. Exp. Mar. Biol. Ecol. 179: 145-169.

Clarke, K.R. 1993. Non-parametric multivariate analyses of changes in community structure. Austral. J. Ecol. 18: 117-143.

Clarke, K.R., P.J. Somerfield \& M.G. Chapman 2006. On resemblance measures for ecological studies, including taxonomic dissimilarities and a zero-adjusted Bray-Curtis coefficient for denuded assemblages. J. Exp. Mar. Biol. Ecol. 330: 55-80.

Crooks, J.A. \& H.S. Khim. 1999. Architectural vs. biological effects of a habitat altering, exotic mussel, Musculista senhousia. J. Exp. Mar. Biol. Ecol. 240: 53-75.

Cruz-Motta, J.J. 2007. Análisis espacial de las comunidades tropicales intermareales asociadas a los litorales rocosos de Venezuela. Cs. Mar. 33: 133-148.

De Grave, S., N.D. Pentcheff, S.T. Ahyong, T. Chan, K.A. Crandall, P.C. Dworschak, D.L. Felder, R.M. Feldmann, C.H. Fransen, L.Y. Goulding, R. Lemaitre, M.E. Low, J.W. Martin, P.K. Ng, C.E. Schweitzer, S.H. Tan, D. Tshudy \& R. Wetzer. 2009. A classification of living and fossil genera of decapod crustaceans. Raffles B. Zool. 21: 1-109.

Díaz, O. \& I. Liñero. 2003. Poliquetos epibiontes de Pinctada imbricata Röding, 1798 (Bivalvia: Pteridae) en el golfo de Cariaco, Venezuela. Interciencia 28: 298-301.

Díaz, J.M. \& M. Puyana. 1994. Moluscos de Caribe colombiano: un catálogo ilustrado. Invemar, Colombia.

Dittel, A., A. Hines, G. Ruiz \& K. Ruffin. 1995. Effects of shallow-water refuge on behavior and density-dependent mortality of juvenile blue crabs in Chesapeake Bay. Bull. Mar. Sci. 57: 902-916.

Eggleston, D.B., L.L. Etherington \& W.E. Elis. 1998. Organism response to habitat patchiness: species and habitat-dependent recruitment of decapod crustaceans. J. Exp. Mar. Biol. Ecol. 223: 111-132.
Eggleston, D.B., W.E. Elis, L.L. Etherington, C.P. Dahlgren \& M.H. Posey. 1999. Organism responses to habitat fragmentation and diversity: Habitat colonization in estuarine macrofauna. J. Exp. Mar. Biol. Ecol. 236: 107-132.

Erlandsson, J., C.D. McQuaid \& V.E. Kostylev. 2005. Contrasting spatial heterogeneity of sessile organisms within mussel (Perna perna L.) beds in relation to topographic variability. J. Exp. Mar. Biol. Ecol. 314: 79-97.

Fuentes, Y. 2012. Macrofauna asociada a Halimeda opuntia en las islas de Cubagua y La Tortuga, Venezuela. Trabajo de grado Licenciatura, Universidad de Oriente, Boca del Río, Venezuela.

Galkin, S.V. \& E.I. Goroslavskaya. 2008. Bottom fauna associated with mussel beds and alvinellid communities in the hydrothermal field at $9^{\circ} \mathrm{N}$ of the East Pacific Rise. Oceanology 48: 509-516.

Gómez, A. 1996. Causas de la fertilidad marina en el nororiente de Venezuela. Interciencia 21: 140-146.

Gosselin, L.A \& F.S. Chia. 1995. Distribution and dispersal of early juvenile snails: effectiveness of intertidal microhabitats as refuges and food sources. Mar. Ecol. Prog. Ser. 128: 213-223.

Guerra-Castro, E., J.J. Cruz-Motta \& J.E. Conde. 2011. Cuantificación de la diversidad de especies incrustantes asociadas a las raíces de Rhizophora mangle L. en el Parque Nacional Laguna de la Restinga. Interciencia 36: 223-230.

Gutiérrez, J.L., C.G. Jones, D.L. Strayer \& O. Iribarne. 2003. Mollusks as ecosystems engineers: the role of the shell production in aquatic environments. Oikos 101: 79-90.

Hendler, G., J.E. Miller, D.L. Pawson \& P.M. Kier. 1995. Sea Stars, Sea Urchins, and Allies: Echinoderms of Florida and the Caribbean. Smithsonian Institution, Washington, EE.UU.

Hernández-Ávila, I., A. Gómez, C. Lira \& L. Galindo. 2007. Benthic decapod crustaceans(Crustacea: Decapoda) from Cubagua Island, Venezuela. Zootaxa 1557: 33-45.

Hernández-Ávila, I. 2010. Ofiuroideos (Echinodermata: Ophiuroidea) de la isla de Cubagua, Venezuela. Tesis Maestría, Instituto Oceanográfico de Venezuela, Cumaná, Venezuela.

Hurlbert, S.H. 1984. Pseudoreplication and the design of ecological field experiments. Ecol. Monogr. 54: 187-211.

Jacobi, C.M. 1987. The invertebrate fauna associated with intertidal beds of the brown mussel Perna perna (L.) from Santos, Brazil. Stud. Neotrop. Fauna E. 22: 37-72.

Jiménez, M., C. Lodeiros \& B. Márquez. 2000. Captación de juveniles de la Madre Perla Pinctada imbricata 
(Röding, 1798) con colectores artificiales en el Golfo de Cariaco, Venezuela. Caribb. J. Sci. 36: 221-226.

Jones, C.G., J.H. Lawton \& M. Shachak. 1997. Positive and negative effects of organisms as physical ecosystem engineers. Ecology 78: 1946-1957.

Licet, B., V. Acosta, A. Prieto \& N. García. 2009. Contribución al conocimiento de los macromoluscos asociados a la Pepitona, Arca zebra (Swainson, 1833), en el banco natural de Chacopata, Península de Araya, Venezuela. Zoot. Trop. 27: 195-203.

Lintas, C. \& R. Seed. 1994. Spatial variation in the fauna associated with Mytilus edulis on a wave-exposed rocky shore. J. Mollusc. St. 60: 165-174.

Lintas, C. \& R. Seed. 1996. Spatial variation in the fauna associated with Mytilus edulis on a wave-exposed rocky shore. J. Mollusc. St. 60: 165-174.

Lodeiros, C., D. Pico, A. Prieto, N. Narváez \& A. Guerra. 2002. Growth and survival of the pearl oyster Pinctada imbricata (Röding 1758) in suspended and bottom culture in the Golfo de Cariaco, Venezuela. Aquacut. Int. 10: 327-338.

McArdle, B.H. \& M.J. Anderson. 2001. Fitting multivariate models to community data: a comment on distance based redundancy analysis. Ecology 82: 290-297.

MacArthur, R.H. \& J.W. MacArthur. 1961. On bird species diversity. Ecology 42: 594-598.

Mackenzie, C., L. Troccoli \& L. León. 2003. History of the Atlantic Pearl-Oyster, Pinctata imbricata, industry in Venezuela and Colombia, with biological and ecological observations. Mar. Fish. Rev. 65: 1-20.

Marcano, J., A. Prieto, A. Lárez, J. Alió \& H. Sanabria. 2005. Crecimiento y mortalidad de Pinctada imbricata (Mollusca: Pteridae) en Guamachito Península de Araya, Estado Sucre, Venezuela. Cienc. Mar. 31: 387-397.

Matias, M.G., A.J. Underwood, D.F. Hochuli \& R.A. Coleman. 2010. Independent effects of patch size and structural complexity on diversity of benthic macroinvertebrates. Ecology 91: 1908-1915.

Newell, R.I. \& E.W. Koch. 2004. Modeling seagrass density and distribution in response to changes in turbidity stemming from bivalve filtration and seagrass sediment stabilization. Estuaries 27: 793-806.

Novoa, D., J. Mendoza, L. Marcano \& J. Cárdenas. 1998. El Atlas pesquero marítimo de Venezuela. MACSARPA y VECEP. Caracas, Venezuela.

Otte, E. 1977. La perla del Caribe, Nueva Cádiz de Cubagua. Boulton, Caracas, Venezuela.

Pech, D., P.L. Ardisson \& E. Bourget. 2001. Settlement of a tropical marine epibenthic assemblage on artificial panels: Influence of substratum heterogeneity and complexity scales. Estuar. Coast. Shelf S. 55: 743-750.
Pielou, E.C. 1975. Ecological diversity. Wiley, Nueva York, EE.UU.

Posey, M.H., C.M. Powel, T.D. Alphin \& E. Townsend. 1999. Use of oyster reefs as habitat for epibenthic fish and decapods. In M. Luckenbach, R. Mann \& J. Wesson (eds.). Oyster reef habitat restoration: A Synopsis and Synthesis of Approaches. Maryland Sea Grant, Virginia, EE.UU.

Prieto, A., O. Ramos, D. Arrieche, J. Villalba \& C. Lodeiros. 2001a. Producción secundaria e índice de condición en Arca zebra (Mollusca: Bivalvia) del Golfo de Cariaco, Venezuela. Rev. Biol. Trop. 49: 599-608.

Prieto, A., L. Ruíz, N. García \& M. Álvarez. 2001b. Diversidad malacológica en una comunidad de Arca zebra (Mollusca: Bivalvia) en Chacopata, Estado Sucre, Venezuela. Rev. Biol. Trop. 49: 591-598.

Ricciardi, A., F. Whoriskey \& J. Rasmusen. 1997. The role of the zebra mussel (Dreissena polymorpha) in structuring macroinvertebrate communities on hard substrata. Can. J. Fish. Aquat. Sci. 54: 2596-2608.

Rodríguez, G. 1980. Crustáceos decápodos de Venezuela. IVIC, Caracas, Venezuela.

Rosenberg, G. 2009. Malacolog 4.1.1: A Database of Western Atlantic Marine Mollusca.

Ruiz, G., A. Hines \& M. Posey. 1993. Shallow water as refuge habitat for fish and crustaceans in nonvegetated estuaries: An example from Chesapeake Bay. Mar. Ecol. Prog. Ser. 99: 1-16.

Russo, A.R. 1987. Role of habitat complexity in mediating predation by the gray damselfish Abudefduf sordidus on epiphytal amphipods. Mar. Ecol. Prog. Ser. 36: 101-105.

Seed, R. 1996. Patterns of biodiversity in the macroinvertebrate fauna associated with mussel patches on rocky shores. J. Mar. Biol. Ass. U.K. 76: 203-210.

Shannon, C.E. 1948. A mathematical theory of communication. Bell System Tech. J. 27: 379-423.

Tagliafico, A., M.S. Rangel \& N. Rago. 2011. Distribución y densidad de dos especies de holoturoideos en la isla de Cubagua, Venezuela. Rev. Biol. Trop. 59: 843-852.

Thiel, M. \& N. Ulrich. 2002. Hard rock versus soft bottom: the fauna associated with intertidal mussel beds on hard bottom along the coast of Chile, and considerations on the functional role of mussel beds. Helgoland Mar. Res. 56: 21-30

Tsuchiya, M. \& M. Nishihira. 1985. Islands of Mytilus edulis as a habitat for small intertidal animals: effect of island size on community structure. Mar. Ecol. Prog. Ser. 25: 71-81.

Tsuchiya, M. \& M. Nishihira. 1986. Islands of Mytilus edulis as a habitat for small intertidal animals: effect of Mytilus age structure on the species composition 
of the associated fauna and community organization. Mar. Ecol. Prog. Ser. 31: 171-178.

Turnipseed, M., K.E. Kinck, R.N. Lipsius, J. Drejer \& C.L. Van Dover. 2004. Diversity in mussel beds at deep-sea hydrothermal vents and cold seeps. Ecol. Lett. 6: 518-523.

Van Dover, C.L. \& J. Trask. 2000. Diversity at deep-sea hydrothermal vent and intertidal mussel beds. Mar. Ecol. Prog. Ser. 195: 169-178.

Villafranca, S. \& M. Jiménez. 2004. Abundancia y diversidad de moluscos asociados al mejillón verde Perna viridis (Bivalvia: Mytilidae) en Guayacán, Estado Sucre, Venezuela. Bol. Inst. Ocenogr. Venezuela, Univ. Oriente 43: 65-76.
Villafranca, S. \& M. Jiménez. 2006. Comunidad de moluscos asociados al mejillón verde Perna viridis (Mollusca: Bivalvia) y sus relaciones tróficas en la costa norte de la Península de Araya, Estado Sucre, Venezuela. Rev. Biol. Trop. 54: 135-144.

Warwick, R.M. \& K.R. Clark. 1995. "New biodiversity" measures reveal a decrease in taxonomic distinctness with increasing stress. Mar. Ecol. Prog. Ser. 129: 301-305.

Welch, J.M., D. Rittschof, T.M. Bullock \& R.B. Forward. 1997. Effects of chemicals cues on settlement behavior of blue crab Callinectes sapidus postlarvae. Mar. Ecol. Prog. Ser. 154: 143-153.

Zoppi, E. 1967. Contribución al estudio de los equinodermos de Venezuela. Acta Biol. Ven. 5: 266-333. 


\section{APÉNDICE 1}

Crustáceos decápodos, moluscos y equinodermos asociados a bancos de P. imbricata y A. zebra en isla de Cubagua, Venezuela

\section{APPENDIX 1}

Decapod crustaceans, mollusks and echinoderms associated to P. imbricata and A. zebra beds in Cubagua Island, Venezuela

\begin{tabular}{|c|c|}
\hline TAXON & Especie \\
\hline \multicolumn{2}{|c|}{ Mollusca, Gastropoda } \\
\hline \multirow[t]{2}{*}{ FISSURELLIDAE } & Diodora cayenensis \\
\hline & Diodora variegata \\
\hline \multirow[t]{4}{*}{ TROCHIDAE } & Calliostoma juvenum \\
\hline & Calliostoma pulchrum \\
\hline & Tegula excavata \\
\hline & Tegula lividumaculata \\
\hline \multirow[t]{2}{*}{ Turbinidae } & Astraea coelata \\
\hline & Turbo castanea \\
\hline \multirow[t]{2}{*}{ NASSARIIDAE } & Nassarius consensus \\
\hline & Nassarius vibex \\
\hline CALyPtraeidaE & Crucibulum auricula \\
\hline \multirow[t]{3}{*}{ Muricidae } & Chicoreus brevifrons \\
\hline & Murex messorius \\
\hline & Murex sp. (juvenil) \\
\hline FASCIOLARIIDAE & Leucozonia nassa \\
\hline Tonnidae & Tonna maculosa \\
\hline Volutidae & Voluta musica \\
\hline Olividae & Olivella nivea \\
\hline \multirow[t]{3}{*}{ MARGINELLIDAE } & Volvarina albolineata \\
\hline & Hyalina sp. \\
\hline & Prunum marginatum \\
\hline Cystiscidae & Persicula interruptolineata \\
\hline \multirow[t]{2}{*}{ Bullidae } & Bulla striata \\
\hline & Bivalvia \\
\hline \multirow[t]{4}{*}{ ARCIDAE } & Anadara brasilensis \\
\hline & Arca imbricata \\
\hline & Barbatia cancellaria \\
\hline & Barbatia dominguensis \\
\hline \multirow[t]{3}{*}{ Chamidae } & Chama florida \\
\hline & Chama macerophylla \\
\hline & Chama sinuosa \\
\hline LUCINIDAE & Codakia orbicularis \\
\hline \multirow[t]{3}{*}{ MYTILIDAE } & Lithophaga aristata \\
\hline & Modiolus americanus \\
\hline & Modiolus squamosus \\
\hline OSTREIDAE & Ostrea equestris \\
\hline ANOMIIDAE & Pododesmus rudis \\
\hline \multirow[t]{3}{*}{ Pectinidae } & Aequipecten lineolaris \\
\hline & Argopecten nucleus \\
\hline & Chlamys sp. \\
\hline Plicatulidae & Plicatula gibbosa \\
\hline
\end{tabular}

\begin{tabular}{|c|c|}
\hline TAXON & Especie \\
\hline Crassatellidae & Eucrassatella speciosa \\
\hline \multicolumn{2}{|c|}{ POLYPLACOPHORA } \\
\hline \multirow[t]{2}{*}{ ACANTHOCHITONIDAE } & Acanthochitona rodea \\
\hline & Acanthochitona venezuelana \\
\hline Chaetopleuridae & Chaetopleura janeirensis \\
\hline \multirow[t]{2}{*}{ ISCHNOCHITONIDAE } & Ischnochiton sp. \\
\hline & Ischnochiton striolatum \\
\hline \multicolumn{2}{|c|}{ Crustacea, Decapoda } \\
\hline SICYONIIDAE & Sicyonia laevigata \\
\hline STENOPODIDAE & Stenopus scutellatus \\
\hline \multirow[t]{5}{*}{ Alpheidae } & Alpheus armillatus \\
\hline & Alpheus beani \\
\hline & Alpheus normanni \\
\hline & Alpheus sp. \\
\hline & Synalpheus cf. minus \\
\hline \multirow[t]{3}{*}{ Palaemonidae } & Cuapetes americanus \\
\hline & Periclimenes iridiscens \\
\hline & Periclimenes yucatanicus \\
\hline \multirow[t]{2}{*}{ HipPOLYTIDAE } & Thor manningi \\
\hline & no id. \\
\hline Processidae & Processa bermudensis \\
\hline \multirow[t]{2}{*}{ Diogenidae } & Clibanarius antillensis \\
\hline & Petrochirus diogenes \\
\hline PAGURIDAE & Pagurus brevidactylus \\
\hline \multirow[t]{2}{*}{ Porcellanidae } & Petrolisthes armatus \\
\hline & Petrolisthes galathinus \\
\hline DROMIIDAE & Moreiradromia antillensis \\
\hline \multirow[t]{2}{*}{ INACHIDAE } & Podochela riseii \\
\hline & Stenorhynchus seticornis \\
\hline \multirow[t]{7}{*}{ MAJIDAE } & Hemus cristulipes \\
\hline & Macrocoeloma trispinosum \\
\hline & Microphrys bicornutus \\
\hline & Mithraculus forceps \\
\hline & Mithrax sp. \\
\hline & Mithrax verrucosus \\
\hline & Stenocionops coelata \\
\hline \multirow[t]{2}{*}{ EPIALTIDAE } & Pitho lherminieri \\
\hline & Tyche emarginata \\
\hline Portunidae & Portunus ordwayi \\
\hline \multirow[t]{3}{*}{ XANTHIDAE } & Cataleptodius floridanus \\
\hline & Micropanope sp. \\
\hline & Platipodiella spectabilis \\
\hline
\end{tabular}


APÉNDICE 1 (Continuación)

Crustáceos decápodos, moluscos y equinodermos asociados a bancos de P. imbricata y A. zebra en isla de Cubagua, Venezuela

APPENDIX 1 (Continued)

Decapod crustaceans, mollusks and echinoderms associated to P. imbricata and A. zebra beds in Cubagua Island, Venezuela

\begin{tabular}{|c|c|c|c|}
\hline TAXON & Especie & TAXON & Especie \\
\hline \multirow[t]{2}{*}{ PANOPEIDAE } & Panopeus herbstii & OPHIACTIDAE & Ophiactis savignyi \\
\hline & Panopeus sp. & AMPHIURIDAE & Ophiostigma isocanthum \\
\hline \multirow[t]{2}{*}{ Pilumnidae } & Pilumnus caribaeus & OPHIOTRICHIDAE & Ophiothrix angulata \\
\hline & Pilumnus dasypodus & & Ophiothrix orstedii \\
\hline PinNotheridaE & Calyptraeotheres hernandezi & & ¿CHINOIDEA \\
\hline \multicolumn{2}{|c|}{ ECHINODERMatA, Asteroidea } & CLYPEASTERIDAE & Clypeaster subdepressus \\
\hline ECHINASTERIDAE & Echinaster echinophorus & ARBACIIDAE & Arbacia punctulata \\
\hline LUIDIIDAE & Luidia alternata & TOXOPNEUSTIDAE & Lytechinus variegatus \\
\hline \multicolumn{2}{|r|}{ OPHIUROIDEA } & \multicolumn{2}{|c|}{ HoLOTHUROIDEA } \\
\hline OPHIURIDAE & Ophiolepis impressa & CUCUMARIIDAE & Aslia surinamensis \\
\hline OPHIOCOMIDAE & Ophiocomella ophiactoides & HOLOTHURIDAE & Holothuria mexicana \\
\hline \multirow[t]{4}{*}{ OPHIODERMATIDAE } & Ophioderma appressa & & Holothuria grisea \\
\hline & Ophioderma brevispina & STICHOPODIDAE & Isostichopus badionotus \\
\hline & Ophioderma cinerea & & \\
\hline & Ophioderma brevicauda & & \\
\hline
\end{tabular}

